\title{
Two-stage two-dimensional guillotine cutting stock problems with usable leftover*
}

\author{
R. Andrade ${ }^{\dagger}$ \\ E. G. Birgin ${ }^{\dagger}$ \\ R. Morabito ${ }^{\ddagger}$
}

July $11,2013^{\S}$

\begin{abstract}
In this study we are concerned with the non-exact two-stage two-dimensional guillotine cutting problem considering usable leftovers, in which stock plates remainders of the cutting patterns (non-used material or trim loss) can be used in the future, if they are large enough to fulfill future demands of items (ordered smaller plates). This cutting problem can be characterized as a residual bin-packing problem because of the possibility of putting back into stock residual pieces, since the trim loss of each cutting/packing pattern does not necessarily represent waste of material depending on its size. Two bilevel mathematical programming models to represent this non-exact two-stage two-dimensional residual bin-packing problem are presented. The models basically consist on cutting/packing the ordered items using a set of plates of minimum cost and, among all possible solutions of minimum cost, choosing one that maximizes the value of the generated usable leftovers. Because of special characteristics of these bilevel models, they can be reformulated as one-level mixed integer programming models. Results of some numerical experiments are presented to show that the models represent appropriately the problem and to illustrate their performances.
\end{abstract}

Key words: Two-stage two-dimensional guillotine cutting, residual bin-packing problem, residual cutting-stock problem, bilevel programming, MIP models, leftovers.

\section{Introduction}

Cutting problems are often found in different industrial processes where paper and aluminium rolls, glass and fibreglass plates, metal bars and sheets, hardboards, pieces of leather and cloth, etc., are cut in order to produce smaller pieces of ordered sizes and quantities. These problems are closely related to packing problems and they basically consist on determining the "best" way to cut large stock objects to produce small ordered items so that one or more objectives are optimized. Cutting and packing problems have been widely studied in the literature in the last decades.

In a number of industrial cutting processes as, for example, in furniture and hardboard companies, the cutting equipment is able to produce only guillotine cuts on the plates (Gilmore \& Gomory 1965, Farley 1983, Yanasse et al. 1991, Carnieri et al. 1994, Morabito \& Arenales 2000, Morabito

*This work was supported by PRONEX-CNPq/FAPERJ E-26/111.449/2010-APQ1, FAPESP (2010/10133-0, 2013/05475-7, and 2013/07375-0), and CNPq.

${ }^{\dagger}$ Department of Computer Science, Institute of Mathematics and Statistics, University of São Paulo, Rua do Matão, 1010, Cidade Universitária, 05508-090, São Paulo, SP, Brazil. e-mail: \{randrade| egbirgin\}@ime.usp.br

${ }^{\ddagger}$ Department of Production Engineering, Federal University of São Carlos, Via Washington Luiz km. 235, 13565905, São Carlos, SP - Brazil. e-mail: morabito@ufscar.br

${ }^{\S}$ Revision made on October 15, 2013, and January 8, 2014. 
\& Belluzzo 2005). A guillotine cut on a plate is a cut from one edge of the plate to the opposite edge, parallel to the remaining edge. In other words, the cut is of guillotine type if when applied to a rectangle it produces two new rectangles. Depending on the cutting equipment, the feasible two-dimensional cutting patterns for the plates can be produced by guillotine cuts in at most two stages. In the first stage, parallel longitudinal (horizontal) guillotine cuts are produced on a plate, without moving it, to produce a set of strips. In the second stage, these strips are pushed, one by one, and the remaining parallel transversal (vertical) guillotine cuts are made on each strip (see Figure 1). If there is no need for additional trimming (i.e. all items have the same height in each strip), the cutting pattern is called exact two-stage guillotine (Figure 1a); otherwise, it is called non-exact (Figure 1b).

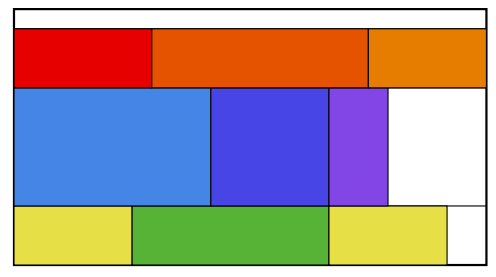

$24 \times 10$

(a)

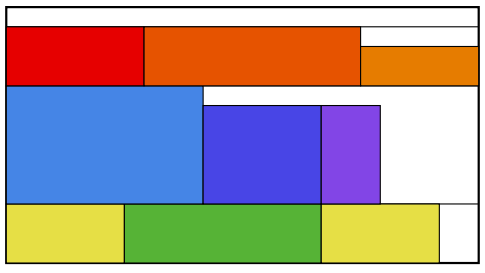

$24 \times 10$

(b)

Figure 1: Two-stage cutting pattern: (a) exact case, (b) non-exact case.

In this study we deal with the non-exact two-stage two-dimensional guillotine cutting problem of how to cut a set of rectangular objects with known sizes and quantities to make exactly a set of rectangular items with specified sizes and demands to be fulfilled. We assume that the assortment of ordered items can be strongly heterogeneous, i.e. the set of items can be characterized by the fact that only very few items are of identical size. We are particularly concerned with the special case of the problem in which the non-used material of the cutting patterns (object remainder or leftover) can be used in the future, if it is large enough to fulfill future items demands. In other words, we consider that the trim loss of a cutting pattern does not necessarily represent waste of material. If this trim loss is of a reasonable size, it can be stocked and used again as input (then called a residual piece or a retail or a leftover) in subsequent cutting processes. Otherwise, if the trim loss is considered too small to be used in the future, it represents material waste and is discarded as scrap. Therefore, the problem is seen as a non-exact two-stage two-dimensional guillotine cutting problem with usable leftovers. Note that the assortment of stock objects of this problem is considered heterogeneous as different leftovers of previous cutting processes are put back into stock.

According to the typology of Wäscher et al. (2007), this cutting problem can be characterized as a "residual bin-packing problem" because of the heterogeneity of the stock objects (based on the possibility of putting back into stock new residual pieces) and the assumption of strongly heterogeneous assortment of small items. Otherwise, if the assortment of items were weakly heterogeneous, the problem would be considered as a "residual cutting-stock problem". In fact, the models presented in this study apply to both problems, since identical items are treated differently than non-identical items in order to avoid symmetric solutions. We observe that the simple objective of minimizing the cost or trim loss of the objects cut may not be appropriate for this problem. The use of leftovers in cutting and packing problems was apparently first discussed in Brown (1971), but studies dealing with this subject began mainly after the work of Dyckhoff (1981). One-dimensional cutting/packing problems that allow the provision of residual pieces have been studied by different 
authors, such as in Roodman (1986), Scheithauer (1991), Gradisar et al. (1999), Sinuany-Stern \& Weiner (1994), Gradisar \& Trkman (2005), Trkman \& Gradisar (2007), Cherri et al. (2009, 2013), Dimitriadis \& Kehris (2009), Cui \& Yang (2010), Gradisar et al. (2011), Bang-Jensen \& Larsen (2012). Examples of applications of one-dimensional cutting problems with usable leftovers were reported in, e.g. the textile industry (Gradisar et al. 1997), the agricultural light aircraft manufacturing (Abuabara \& Morabito 2009), and the wood-processing industry (Koch et al. 2009). To the best of our knowledge, all studies reported in the literature focused in one-dimensional residual bin-packing problems, the exception being the recent published paper (Andrade et al. 2014) that deals with two-dimensional non-guillotine cutting problems with usable leftovers. We are not aware of other studies dealing with residual bin-packing problems involving two or more dimensions.

The paper is organized as follows. In Section 2 we present two MIP models for the two-stage two-dimensional bin-packing problem without considering leftovers. These models are highly based on models introduced in Lodi \& Monaci (2003) for the two-stage two-dimensional knapsack problem. Then, in Section 3, we present two bilevel models for the two-stage two-dimensional residual binpacking problem and their one-level MIP reformulations. In Section 4 we report and analyse the numerical results obtained by solving the models using the branch-and-cut method of CPLEX. Finally, in Section 5 we present concluding remarks and discuss perspectives for future research.

\section{Two-stage bin-packing models without leftovers}

In this section we present two MIP models for the non-exact two-stage two-dimensional bin-packing problem without considering leftovers. These models are straightforward extensions of models M1 and M2 introduced in Lodi \& Monaci (2003) for the two-stage two-dimensional knapsack problem. Other two-stage two-dimensional knapsack models could be considered as, for example, the ones discussed in Silva et al. (2010), Furini \& Malaguti (2013). Only a few studies are found in the literature dealing with two-stage two-dimensional bin-packing problems. Most of the studies are concerned with either the two-stage two-dimensional knapsack problem or the two-stage twodimensional cutting stock problem. An example is the method developed by Gilmore \& Gomory (1965), based on the simplex method with a column generation procedure to generate two-stage cutting patterns. This procedure involves two phases. In the first phase cutting patterns are determined for each longitudinal strip, while the second phase decides how many times each strip should be used. This method works well if the assortment of ordered items is weakly heterogeneous, i.e. the small items can be grouped into relatively few classes and the quantity of items in each class is "sufficiently" large, as it is the case of two-stage two-dimensional cutting stock problems (Wäscher et al. 2007).

Solution approaches for two-stage two-dimensional cutting stock problems based on two phases are common in the literature, as for example in Farley (1983), Riehme et al. (1996), Hifi (1997), Morabito \& Garcia (1998), Yanasse \& Katsurayama (2005). For the case in which the set of items has few items of identical size, authors have proposed alternatives for the rounding of relaxed solutions of the simplex method (Wäscher \& Gau 1996, Poldi \& Arenales 2006) or greedy heuristics combined with column generation procedures (Hinxman 1980, Poldi \& Arenales 2009) that may not work well for two-stage two-dimensional bin-packing problems. Other studies dealing with stage guillotine cutting problems can be found in Beasley (1985), Hadjiconstantinou \& Christofides (1995), Morabito \& Arenales (1996), Riehme et al. (1996), Hifi (1997), Hifi \& Roucairol (2001), Lodi \& Monaci (2003), Cui et al. (2005), Belov \& Scheithauer (2006), Cintra et al. (2008), Silva et al. (2010), Morabito \& Pureza (2010), Cui (2013), Cui \& Huang (2012), Cui \& Zhao (2013), Furini \& Malaguti (2013), Furini et al. (2012), Mrad et al. (2013), Alvarez-Valdes et al. (2002), Lodi et al. (2002), Macedo et al. (2010). 


\subsection{Two-stage bin-packing model $\mathcal{M}_{1}$}

Let us consider $p$ large rectangular objects, each object $\ell$ with width $W_{\ell}$, height $H_{\ell}$ and cost per unit of area $c_{\ell}(\ell=1, \ldots, p)$ and $n$ small rectangular items, each item $i$ with width $w_{i}$ and height $h_{i}(i=1, \ldots, n)$. The non-exact two-stage two-dimensional bin-packing problem can be defined as the problem of packing (cutting) all $n$ items into (from) a chosen subset of objects, so that the obtained packing (cutting) pattern for each object is feasible (the packed items do not overlap and fit inside the object according to a non-exact two-stage guillotine pattern) and the cost of the used objects is minimized. We consider only the case in which the first stage cuts on the plate are horizontal, i.e., they are parallel to the object width. No item rotations are allowed and there are no other constraints related to the positioning of the items within the objects.

Without loss of generality, we assume that $h_{1} \geq h_{2} \geq \cdots \geq h_{n}$. We also assume that the cuts on the objects are infinitely thin; otherwise, we consider that the saw thickness was added to the dimensions of the objects and items, without loss of generality (Gilmore \& Gomory 1965, Morabito \& Arenales 2000). Moreover, we assume that all dimensions of the objects and items, as well as the objects unit costs, are integer numbers. This is not a very restrictive assumption to deal with problem instances in practice since, due to the finite precision of the cutting and measuring tools and due to the finite precision used in any currency considered to define the objects' costs, they can be easily satisfied by a change of scale. The model presented below can be seen as a simple extension of the two-stage knapsack model M1 introduced in Lodi \& Monaci (2003, p.261) to deal with the two-stage bin-packing problem. The original objective function of the model is modified in order to appropriately consider more than one object. For this, we define the binary variables $u_{\ell}(\ell=1, \ldots, p)$, which indicate if object $\ell$ is used or not:

$$
u_{\ell}= \begin{cases}1, & \text { if object } \ell \text { is used } \\ 0, & \text { otherwise }\end{cases}
$$

The other binary variables $x_{i k \ell}(k=1, \ldots, n, i=k, \ldots, n, \ell=1, \ldots, p)$ indicate the object and strip from which the item is cut:

$$
x_{i k \ell}= \begin{cases}1, & \text { if item } i \text { is cut from strip } k \text { of object } \ell \\ 0, & \text { otherwise. }\end{cases}
$$

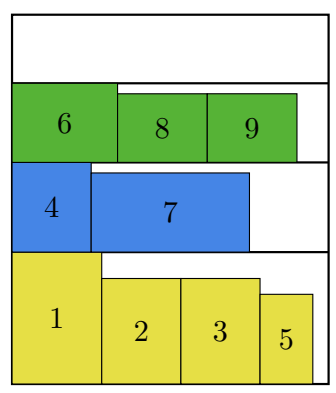

Figure 2: Illustration of the concept of shelves.

Following the terminology used in Lodi \& Monaci (2003), given an object $\ell$, a shelf is defined as a strip of the object with width $W_{\ell}$ and height equal to the height of the highest item packed (cut) in (from) it. It is assumed that all items in a shelf have their bottom (inferior side) on the shelf floor. The roof of the shelf, determined by the top (superior side) of the item of largest height, 
defines the floor of the next shelf. The shelf concept is illustrated in Figure 2. In this figure, there is one object and three shelves: 1, 4, and 6 . Items 1,2,3 and 5 are in shelf 1 , items 4 and 7 are in shelf 4 , and items 6,8 and 9 are in shelf 6 . Note that the number of each shelf is defined as the number of the first item packed in it. In the model presented below, we consider that we can have up to $n$ shelves, each one defined by an item. We say that a shelf $k$ is open (or used) if item $k$ is the smallest-index item assigned to (or packed in) the shelf. In this case, if item $k$ is on shelf $k$ and shelf $k$ is assigned to object $\ell$, we have $x_{k k \ell}=1$. Note that any optimal two-stage cutting pattern has an equivalent cutting pattern where the item of largest height in each shelf is the first item placed to the left of the shelf (as depicted in Figure 2). A feasible two-stage guillotine cutting pattern is composed of shelves and each item allocated to a shelf is cut in at most two stages (plus the trimming). A model named $\mathcal{M}_{1}$ for the non-exact two-stage bin-packing problem (without leftovers) is given by:

$$
\begin{aligned}
& \operatorname{Min}_{u, x} \quad \sum_{\ell=1}^{p} c_{\ell} W_{\ell} H_{\ell} u_{\ell} \\
& \begin{array}{ll}
\text { s.t. } & \sum_{k=1}^{n} h_{k} x_{k k \ell} \\
& \sum_{i=k+1}^{n} w_{i} x_{i k \ell}
\end{array} \\
& \begin{array}{l}
\sum_{i=k+1}^{p} w_{i} x_{i k \ell} \\
\sum_{\ell=1}^{p} \sum_{k=1}^{i} x_{i k \ell}
\end{array} \\
& \sum_{\ell=1}^{\ell=1} x_{k+1, k+1, \ell} \\
& \sum_{\ell=1}^{p} \sum_{i=k+2}^{\alpha_{j}} x_{i, k+1, \ell} \\
& u_{\ell} \\
& x_{i k \ell}
\end{aligned}
$$

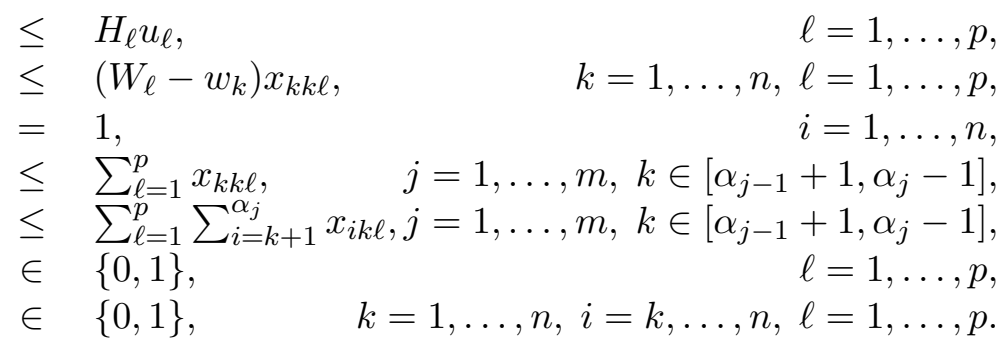

The objective function (3) minimizes the total cost of the objects used (note that, if $c_{\ell}=1$ for all $\ell,(3)$ minimizes the total object area cut). Constraint (4) ensures that, for each used object, the sum of the heights of the open shelves is not greater than the object height, and that open shelves are attributed to used objects only. Constraint (5) ensures that, for each object, the sum of the widths of the items allocated to each shelf is not greater than the object width, and that an item can be allocated to a shelf only if the shelf is open. Constraint (6) ensures that the demand of each item is met. Constraints (7) and (8) are redundant symmetry-breaking constraints and refer to identical items. Without loss of generality, we assume that identical items (items with the same width and height) are numbered consecutively. We also assume that there are $m$ different types of items and we define $\alpha_{0} \equiv 0$ and $\alpha_{j}$ as last index of items of the $j$-th type. It means that the indices of items of type $j$ range from $\alpha_{j-1}+1$ to $\alpha_{j}$. Symmetry-breaking constraint (7) says that an item that is not the first of its type can open a shelf only if the previous item (of the same type) opens a shelf too. Symmetry-breaking constraint (8) says that if two consecutive items $k$ and $k+1$ of the $j$-th type open a shelf, the number of items of type $j$ on shelf $k+1$ must be less than or equal to the number of items of type $j$ on shelf $k$. Constraints (9) and (10) define the domain of variables $u_{\ell}$ and $x_{i k \ell}$.

\subsection{Two-stage bin-packing model $\mathcal{M}_{2}$}

In Lodi \& Monaci (2003, p.262) another model for the non-exact two-stage two-dimensional knapsack problem was presented, named M2, which considers identical items as items of the same group or type. In the following we present a slightly modified version of this model to solve the two-stage two-dimensional bin-packing problem. The model assumes that there are $n$ items of $m$ different types. Items of type $i$ have width $\bar{w}_{i}$ and height $\bar{h}_{i}$. We assume, without loss of generality, that $\bar{h}_{1} \geq \bar{h}_{2} \geq \cdots \geq \bar{h}_{m}$. The demanded quantity of items of type $i$ is given by $b_{i}$. The additional model parameters (that can be easily computed from the other ones) are: $\alpha_{i}(i=0, \ldots, m)$ and $\beta_{k}$ 
$(k=1, \ldots, n)$, with $\alpha_{0} \equiv 0, \alpha_{i} \equiv \sum_{s=1}^{i} b_{s}(i=1, \ldots, m)$ (that coincides with the definition introduced in the previous subsection), and $\beta_{k} \equiv \min \left\{i \mid \alpha_{i} \geq k\right\}(k=1, \ldots, n)$. Note that: (i) any item of type $i$ can be packed in any shelf in the interval [1, $\left.\alpha_{i}\right]$, i.e. $\alpha_{i}$ indicates the highest shelf index to allocate items of type $i$ and (ii) indices in the interval $\left[\alpha_{i-1}+1, \alpha_{i}\right]$ can be interpreted as the indices of the shelves characterized by items of type $i$. Moreover, each shelf $k$ can pack items of types $\left[\beta_{k}, m\right]$, i.e. parameter $\beta_{k}$ can be seen as the index of the item type of largest height (lowest index) that can be allocated in shelf $k$. In other words, $\beta_{k}$ is the index of the item type that defines shelf $k$. Note that there is a shelf for each item.

The former variables $x_{i k \ell}(k=1, \ldots, n, i=k, \ldots, n, \ell=1, \ldots, p)$ are also used in this model, but with a slightly different meaning. Now they are integer (instead of binary) and relate items and shelves in the following way:

$$
x_{i k \ell}= \begin{cases}\text { quantity of items of type } i \text { allocated to shelf } k \text { of object } \ell, & \text { if } i \neq \beta_{k}, \\ \text { quantity of additional items of type } i \text { (other than the one } & \\ \text { that defines the shelf) allocated to shelf } k \text { of object } \ell, & \text { if } i=\beta_{k},\end{cases}
$$

with $i=1, \ldots, m, k \in\left[1, \alpha_{i}\right], \ell=1, \ldots, p$. The model also considers the binary variables $q_{k \ell}$ $(k=1, \ldots, n, \ell=1, \ldots, p)$, which indicate if the shelf is open (used) or not:

$$
q_{k \ell}= \begin{cases}1, & \text { if shelf } k \text { of object } \ell \text { is open, } \\ 0, & \text { otherwise }\end{cases}
$$

The remaining model parameters and variables are the same as the model of the previous section. The second model for the two-stage bin-packing problem (without leftovers), named $\mathcal{M}_{2}$, is given by:

$$
\begin{aligned}
& \operatorname{Min}_{u, x, q} \sum_{\ell=1}^{p} c_{\ell} W_{\ell} H_{\ell} u_{\ell} \\
& \begin{array}{ll}
\text { s.t. } & \sum_{k=1}^{n} \bar{h}_{\beta_{k}} q_{k \ell} \\
& \sum_{i=\beta_{k}}^{m} \bar{w}_{i} x_{i k \ell}
\end{array} \\
& \sum_{\ell=1}^{p}\left(\sum_{k=1}^{\alpha_{i}} x_{i k \ell}+\sum_{k=\alpha_{i-1}+1}^{\alpha_{i}} q_{k \ell}\right) \\
& \sum_{\ell=1}^{p} q_{k \ell} \\
& \sum_{\ell=1}^{p} q_{k+1, \ell} \\
& \sum_{\ell=1}^{p} x_{i, k+1, \ell} \\
& x_{i k \ell} \\
& u_{\ell} \\
& x_{i k \ell} \\
& q_{k \ell}
\end{aligned}
$$

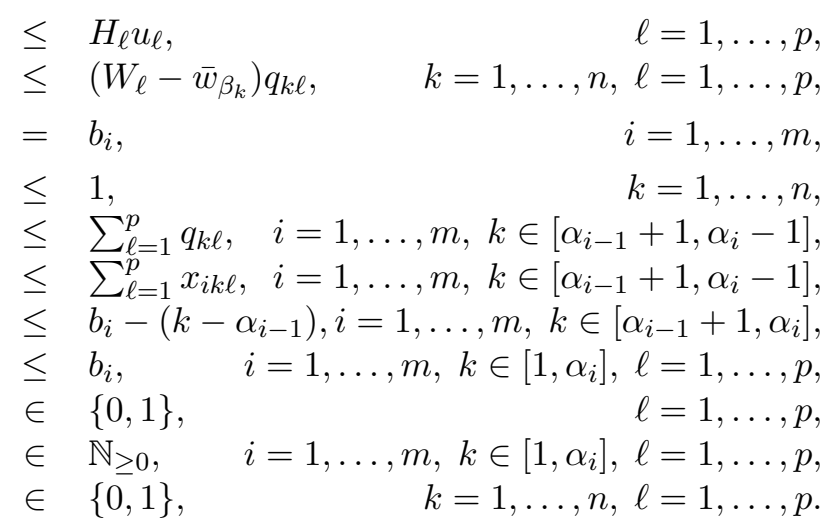

Constraint (14) ensures that, for each object, the sum of the heights of the open shelves is less than or equal to the object height and that shelves are opened in used objects only. Constraint (15) ensures that, for each object, the sum of the widths of the items allocated to each shelf is not greater than the object width. Constraint (16) ensures that the demand of each item is met. Constraint (17) ensures that each shelf can be opened only once (i.e. we cannot have a shelf $k$ opened in two different objects). Constraints (18) and (19) are redundant symmetry-breaking constraints equivalent to (7) and (8) from model $\mathcal{M}_{1}$. Constraint (20) is a redundant constraint considered in Lodi \& Monaci (2003) to improve the quality of the lower bounds of the LP relaxation of the model (removing the integrality constraints). Constraints (21-23) define the domain of the variables.

Model $\mathcal{M}_{1}$ has $2 p$ continuous variables, $2 p+n p / 2+n^{2} p / 2$ binary variables, and $p+n p+3 n-2 m$ constraints. Model $\mathcal{M}_{2}$ has $2 p$ continuous variables, $2 p+n p$ binary variables, $p \sum_{k=0}^{m} \alpha_{k}$ integer 
variables, and $5 p+n p+4 n-m+p \sum_{k=0}^{m} \alpha_{k}$ constraints. Note that both models have the same quantity of continuous variables, but differ in terms of the number of binary and integer variables. Model $\mathcal{M}_{1}$ has $O\left(n^{2} p\right)$ binary variables, while model $\mathcal{M}_{2}$ has only $O(n p)$. Model $\mathcal{M}_{1}$ does not have integer variables, whereas model $\mathcal{M}_{2}$ has $p \sum_{k=0}^{m} \alpha_{k}$. Analyzing $\sum_{k=0}^{m} \alpha_{k}$, we have the following extreme cases: (i) if $m=1$, then $\sum_{k=0}^{m} \alpha_{k}=n$, (ii) if $m=n$ and $\bar{h}_{1}>\bar{h}_{2}>\cdots>\bar{h}_{m}$, then $\sum_{k=0}^{m} \alpha_{k}=n(n+1) / 2$, (iii) if $m=n$ and $\bar{h}_{1}=\bar{h}_{2}=\cdots=\bar{h}_{m}$, then $\sum_{k=0}^{m} \alpha_{k}=m n=n^{2}$. In this way, in the worst case, model $\mathcal{M}_{2}$ has $O\left(n^{2} p\right)$ integer variables. Considering the constraints, we observe that model $\mathcal{M}_{1}$ has $O(n p)$ constraints. Model $\mathcal{M}_{2}$ has $O\left(n^{2} p\right)$ constraints in the worst case. Note that in cases where $m$ is a relatively small number compared to $n$, model $\mathcal{M}_{2}$ should have an amount of integer variables and constraints proportional to $n p$. Therefore, as the ratio $m / n$ decreases, we expect that model $\mathcal{M}_{2}$ becomes easier to solve than model $\mathcal{M}_{1}$.

\section{Two-stage residual bin-packing models $\mathcal{M}_{1}^{L}$ and $\mathcal{M}_{2}^{L}$}

In this section, we present models for the two-stage bin-packing problem considering leftovers. While, in the two-dimensional scenario, leftovers can be defined in several ways, we arbitrarily consider leftover as any trim loss of height not smaller than $d_{\min }$, obtained after producing the first-stage horizontal cuts in the object. On the one hand, it appears as a natural choice to separate the leftovers in the first stage of the cutting procedure, considering any other residual piece of the second-stage cuts and the trimming as scrap. This decision appears to be in agreement with a low-cost strategy to deal with leftovers in the production and stocking environment. On the other hand, paying the price of dealing with more complicated models, any other practical definition of leftover can be modeled and tackled in a way similar to the one presented here.

We look for a solution that minimizes the costs of the used objects and, among all minimum cost solutions, we look for one that maximizes the sum of the values of the leftovers. As illustrative example, consider an instance with $p=2$ identical objects with $W_{1}=W_{2}=H_{1}=H_{2}=9$ and $c_{1}=c_{2}=1$, and $n=8$ items with $w_{1}=\cdots=w_{4}=4, w_{5}=\cdots=w_{8}=3, h_{1}=\cdots=h_{6}=4$, and $h_{7}=h_{8}=2$. Figures $3(\mathrm{a}-\mathrm{b})$ represent two different feasible solutions (to models $\mathcal{M}_{1}$ and $\mathcal{M}_{2}$ that do not consider leftovers) with cost 162. Since the sum of the areas of the eight demanded items is larger than the area of a single object, at least two objects are needed to cut the items and, hence, both depicted feasible solutions are optimal. However, there is a feature that differentiate these two optimal solutions and that is not being captured by models $\mathcal{M}_{1}$ and $\mathcal{M}_{2}$. If we consider $d_{\text {min }}$ equal to the smallest height of a demanded item, i.e. $d_{\min }=2$, we have that the solution depicted on Figure 3(b) has a leftover in one of its objects, while the solution depicted on Figure 3(a) has no (usable) leftovers.
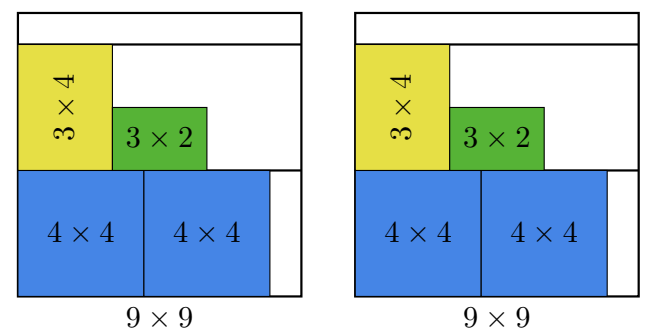

(a)
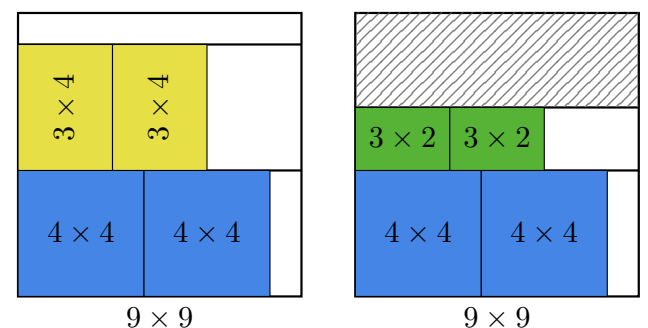

(b)

Figure 3: Illustration of the concept of leftovers in the two-stage bin-packing problem. 
A natural modeling approach for considering leftovers would be to consider a bilevel mathematical programming problem. A bilevel mathematical programming problem (see, for example, (Dempe 2002)) is an optimization problem that maximizes or minimizes an objective function with some of the problem variables restricted to be a solution to another optimization problem. The two models presented below for the two-stage residual bin-packing problem are bilevel models with integer and continuous variables and linear objective functions and constraints. Before presenting the models, we explain how to model the leftovers. Let us consider $s_{\ell}$ as the height of the trim loss of object $\ell=1, \ldots, p$. The trim loss of object $\ell$ is considered as a leftover (i.e. a residual piece) if its height $s_{\ell}$ is such that $s_{\ell} \geq d_{\min }$, where $d_{\min } \geq 0$ is a given parameter. If the trim loss is not a leftover, it is considered as a waste (i.e. a scrap) and its value is null. Therefore, to model the leftovers area we define function $\bar{s}\left(s_{\ell}\right)$ as:

$$
\bar{s}\left(s_{\ell}\right)= \begin{cases}W_{\ell} s_{\ell}, & \text { if } s_{\ell} \geq d_{\min } \\ 0, & \text { otherwise }\end{cases}
$$

The value of the leftover is defined as its area times its unit area value, given by $\bar{c}_{\ell}>0$. It would be reasonable to consider $\bar{c}_{\ell} \equiv c_{\ell}$ for $\ell=1, \ldots, p$, i.e. to set the unit area value of a leftover as the unit area cost of the corresponding object. Note that, in practice, if the unit area value of the leftovers is independent of the objects from which they were cut, one may simply consider $\bar{c}_{\ell}=1$ for all $\ell$.

We can use (25) as constraints of a MIP by applying the big- $M$ technique. To simplify the explanation, we first present a bilevel model based on model $\mathcal{M}_{1}$ to represent the two-stage residual bin-packing problem. Then we discuss how to use (25) as MIP constraints, as well as the remaining details of the model:

$$
\begin{aligned}
& \operatorname{Max}_{z, T} \quad \sum_{\ell=1}^{p} \bar{c}_{\ell} T_{\ell} \\
& \text { s.t. } \quad d_{\min } \leq s_{\ell}+M_{\ell} z_{\ell} \\
& s_{\ell} \leq d_{\min }+M_{\ell}\left(1-z_{\ell}\right), \\
& T_{\ell} \quad \leq s_{\ell} W_{\ell}+\bar{M}_{\ell} z_{\ell}, \\
& T_{\ell} \leq \bar{M}_{\ell}\left(1-z_{\ell}\right), \\
& T_{\ell} \quad \geq 0, \\
& z_{\ell} \quad \in\{0,1\} \text {, } \\
& (u, x, s) \in \underset{u^{\prime}, x^{\prime}, s^{\prime}}{\operatorname{argmin}} \sum_{\ell=1}^{p} c_{\ell} W_{\ell} H_{\ell} u_{\ell}^{\prime} \\
& \text { s.t. } \sum_{k=1}^{u^{\prime}, x^{\prime}, s^{\prime}} h_{k} x_{k k \ell}^{\prime}+s_{\ell}^{\prime}=H_{\ell} u_{\ell}^{\prime} \\
& \sum_{i=k+1}^{n} w_{i} x_{i k \ell}^{\prime} \leq\left(W_{\ell}-w_{k}\right) x_{k k \ell}^{\prime},
\end{aligned}
$$

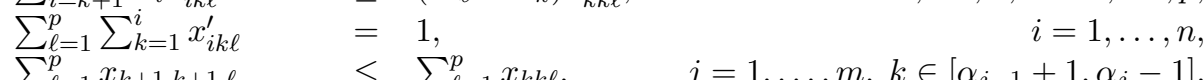

$$
\begin{aligned}
& \sum_{\ell=1}^{x_{k+1, k+1, \ell}} \leq \sum_{\ell=1}^{x_{k k \ell},} \quad j=1, \ldots, m, k \in\left[\alpha_{j-1}+1, \alpha_{j}-1\right], \\
& \begin{array}{r}
\sum_{\ell=1}^{p} \sum_{i=k+2}^{\alpha_{j}} x_{i, k+1, \ell} \leq \sum_{\ell=1}^{p} \sum_{i=k+1}^{\alpha_{j}} x_{i k \ell}, j=1, \ldots, m, k \in\left[\alpha_{j-1}+1, \alpha_{j}-1\right], \\
\ell=\{0,1\},
\end{array} \\
& x_{i k \ell}^{\prime} \in\{0,1\}, \\
& s_{\ell}^{\prime} \geq 0 \text {, }
\end{aligned}
$$

The inferior level problem (33-41) ensures that the total area cost of the objects used is minimized. The superior level problem (26-32) ensures that the sum of the values of the leftover areas generated from the objects cut is maximized, considering as feasible points the solutions to the inferior level problem that satisfy constraints $(27-32)$. Variables $s_{\ell}(\ell=1, \ldots, p)$ indicate the height of the leftover of each object. Constraints (27-32), together with the objective function (26), formulate $\bar{s}\left(s_{\ell}\right)$ defined in (25) by means of the big- $M$ technique. For this, we need to define parameters $M_{\ell}$ and $\bar{M}_{\ell}$ such that $M_{\ell} \geq H_{\ell}$ and $\bar{M}_{\ell} \geq H_{\ell} W_{\ell}(\ell=1, \ldots, p)$, and we use a binary variable $z_{\ell}$ for each object $\ell$. If the height $s_{\ell}$ of the leftover of object $\ell$ is smaller than $d_{\min }$, the corresponding $z_{\ell}$ is forced to assume value one by $(27)$. In this case, by $(30-31)$, we have $T_{\ell}=0$. 
If $s_{\ell}$ is greater than $d_{\min }$, the corresponding $z_{\ell}$ is forced to be zero by (28) and, by (29-31) and the objective function (26), $T_{\ell}$ assumes the value of the leftover area. If $s_{\ell}=d_{\min }, z_{\ell}$ may assume value zero or one and, by the objective function (26), $T_{\ell}$ assumes the value of the leftover area. Constraint (33) ensures that $(u, x, s)$ is a solution to the inferior level problem, in which the cost of the objects used to satisfy the demand is minimized. The inferior level problem composed by the objective function (33) and constraints (34-41) is essentially model $\mathcal{M}_{1}$ defined in (3-10), except for constraint (34), that differs of (4) by the addition of the term $s_{\ell}^{\prime}$ that incorporates the leftover height of each object, and constraint (41) that says that the leftovers heights are non-negative quantities. Constraint (34) ensures that the sum of the heights of the open shelves of an object plus the height of its leftover must be equal to the height of the object.

It should be noted that the use of an object could be prioritized in the model by simply nulling its unit area cost $c_{\ell}$. This could be interesting to prioritize the use of leftovers (generated in previous periods) in the cutting pattern generation in order to avoid buying new objects while leftovers accumulate in stock. This situation could be accomplished by setting $c_{\ell}=0$, to prioritize the use of object $\ell$, while keeping in $\bar{c}_{\ell}$ the original unit area cost of object $\ell$, to correctly represent the value of its leftover.

In the following we shortly present the second bilevel model for the two-stage residual binpacking problem based on model $\mathcal{M}_{2}$. The explanation of this model follows the same steps of the presentation of the previous bilevel model based on model $\mathcal{M}_{1}$. The model is given by:

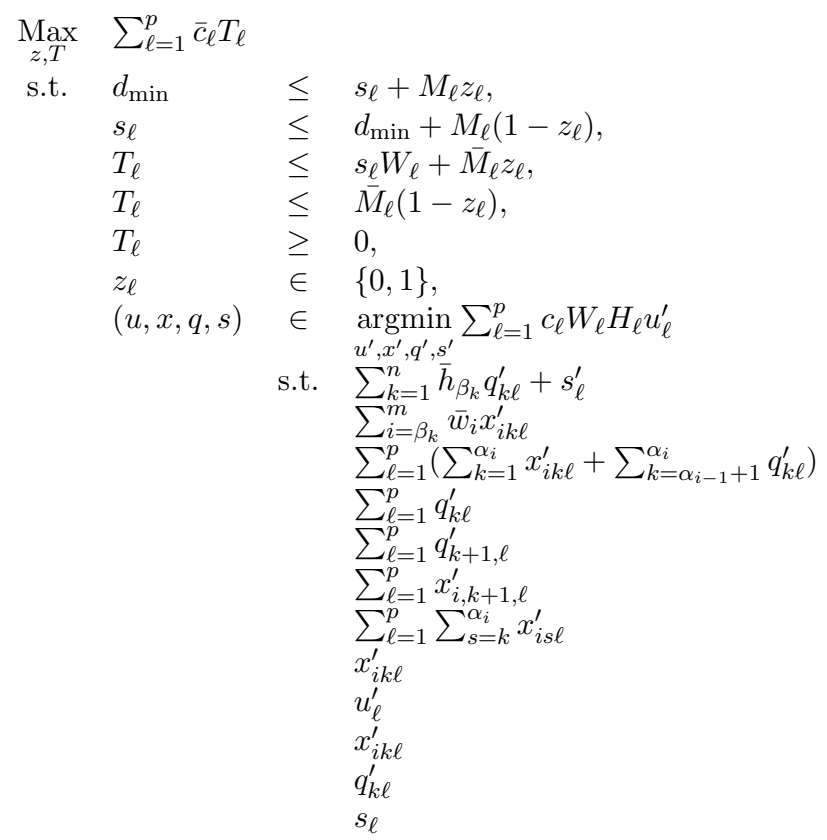

\subsection{MIP reformulations}

A technique commonly applied to solve bilevel mathematical programming models is, when possible, to reformulate the model as an equivalent one-level model. In the following we discuss a simple way to reformulate the bilevel models of previous section as MIP models. For instance, for model $\mathcal{M}_{1}$, considering that the objective function (33) of the inferior level problem assumes only integer values (variables $u_{\ell}, \ell=1, \ldots, p$, are binary and the objects unit costs $c_{\ell}, \ell=1, \ldots p$, are integer numbers by hypothesis) and that it is independent of the variables of the superior level problem, the bilevel model based on model $\mathcal{M}_{1}$ and given by $(26-41)$ can be reformulated as the following MIP: 


$$
\begin{aligned}
& \operatorname{Min}_{z, T, u, x, s} F(T, u) \equiv \sum_{\ell=1}^{p} c_{\ell} W_{\ell} H_{\ell} u_{\ell}-\left(\sum_{\ell=1}^{p} \bar{c}_{\ell} T_{\ell}\right) /\left(\sum_{\ell=1}^{p} \bar{c}_{\ell} W_{\ell} H_{\ell}\right) \\
& \text { s.t. } d_{\min } \leq s_{\ell}+M_{\ell} z_{\ell} \text {, } \\
& s_{\ell} \quad \leq d_{\min }+M_{\ell}\left(1-z_{\ell}\right), \quad \ell=1, \ldots, p, \\
& T_{\ell} \quad \leq s_{\ell} W_{\ell}+\bar{M}_{\ell} z_{\ell}, \quad \ell=1, \ldots, p, \\
& T_{\ell} \quad \leq \bar{M}_{\ell}\left(1-z_{\ell}\right), \quad \ell=1, \ldots, p, \\
& T_{\ell} \quad \geq 0, \quad \ell=1, \ldots, p, \\
& z_{\ell} \quad \in\{0,1\}, \quad \ell=1, \ldots, p, \\
& \sum_{k=1}^{n} h_{k} x_{k k \ell}+s_{\ell} \quad=H_{\ell} u_{\ell}, \quad \ell=1, \ldots, p, \\
& \sum_{i=k+1}^{n} w_{i} x_{i k \ell} \quad \leq\left(W_{\ell}-w_{k}\right) x_{k k \ell}, \quad k=1, \ldots, n, \ell=1, \ldots, p, \\
& \sum_{\ell=1}^{p} \sum_{k=1}^{i} x_{i k \ell} \quad=1, \quad i=1, \ldots, n, \\
& \sum_{\ell=1}^{p} x_{k+1, k+1, \ell} \leq \sum_{\ell=1}^{p} x_{k k \ell}, \quad j=1, \ldots, m, k \in\left[\alpha_{j-1}+1, \alpha_{j}-1\right], \\
& \sum_{\ell=1}^{p} \sum_{i=k+2}^{\alpha_{j}} x_{i, k+1, \ell} \leq \sum_{\ell=1}^{p} \sum_{i=k+1}^{\alpha_{j}} x_{i k \ell}, j=1, \ldots, m, k \in\left[\alpha_{j-1}+1, \alpha_{j}-1\right] \text {, } \\
& u_{\ell} \\
& x_{i k \ell} \\
& s_{\ell} \\
& \in\{0,1\}, \quad \ell=1, \ldots, p \\
& \in\{0,1\}, \quad k=1, \ldots, n, i=k, \ldots, n, \ell=1, \ldots, p, \\
& \geq 0 \text {, }
\end{aligned}
$$

Note that the objective function $F(T, u)$ in $(62)$ is composed by the objective functions of the superior and inferior levels (26) and (33), respectively, where the superior level objective function (26) was normalized in such a way as to assume only values in the interval $[0,1)$. The value of $F(T, u)$ is integer at feasible points with no leftovers, while it is rational at feasible points with leftovers. Using the value of $F(T, u)$ at a feasible point, it is easy to obtain the corresponding values of $(26)$ and $(33)$ as $\lceil F(T, u)\rceil$ and $(\lceil F(T, u)\rceil-F(T, u)) \sum_{\ell=1}^{p} \bar{c}_{\ell} W_{\ell} H_{\ell}$, respectively. Note that constraints (63-76) correspond exactly to constraints (27-32) and (34-41). From now on, we call the model given by $(62-76)$ as model $\mathcal{M}_{1}^{L}$. Regarding the size of the model, it has $2 p$ continuous variables, $2 p+n p / 2+n^{2} / 2$ binary variables, and $7 p+n p+3 n-2 m$ constraints.

Following the same steps to define model $\mathcal{M}_{1}^{L}$, we combine (62) with constraints $(43-48,50-61)$ and define model $\mathcal{M}_{2}^{L}$ given by:

$$
\begin{aligned}
& \operatorname{Min}_{z, T, u, x, s} F(T, u) \equiv \sum_{\ell=1}^{p} c_{\ell} W_{\ell} H_{\ell} u_{\ell}-\left(\sum_{\ell=1}^{p} \bar{c}_{\ell} T_{\ell}\right) /\left(\sum_{\ell=1}^{p} \bar{c}_{\ell} W_{\ell} H_{\ell}\right) \\
& \text { s.t. } \quad d_{\min } \\
& s_{\ell} \\
& T_{\ell} \\
& T_{\ell} \\
& T_{\ell} \\
& \begin{array}{l}
\sum_{\ell=1}^{n} \bar{h}_{\beta_{k}} q_{k \ell}+s_{\ell} \\
\sum_{i=\beta_{k}}^{m} \bar{w}_{i} x_{i k \ell}
\end{array} \\
& \sum_{\ell=1}^{p}\left(\sum_{k=1}^{\alpha_{i}} x_{i k \ell}+\sum_{k=\alpha_{i-1}+1}^{\alpha_{i}} q_{k \ell}\right) \\
& \sum_{\ell=1}^{p} q_{k \ell} \\
& \sum_{\ell=1}^{p} q_{k+1, \ell} \\
& \sum_{\ell=1}^{p} x_{i, k+1, \ell} \\
& x_{i k \ell} \\
& u_{\ell} \\
& x_{i k \ell} \\
& q_{k \ell} \\
& s_{\ell}
\end{aligned}
$$

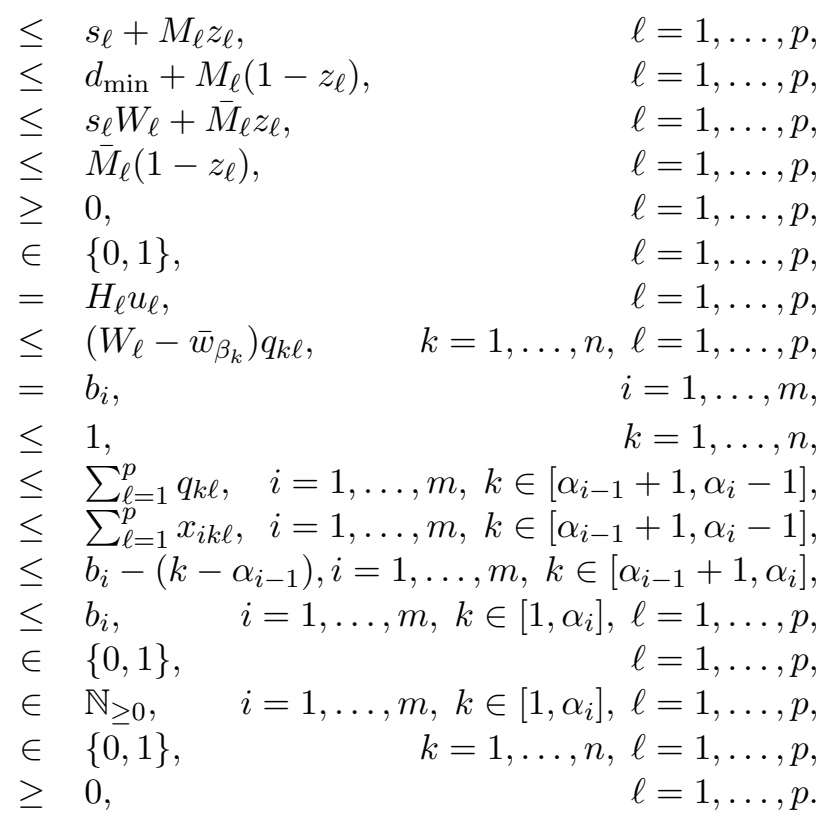

Model $\mathcal{M}_{2}^{L}$ has $2 p$ continuous variables, $2 p+n p$ binary variables, and $7 p+4 n+n p-m+p \sum_{i=1}^{m} \alpha_{i}$ constraints. 
Models $\mathcal{M}_{1}^{L}$ and $\mathcal{M}_{2}^{L}$ deal with the non-exact two-stage two-dimensional guillotine cutting problem. It should be noted that these models can be easily adapted to treat the exact case of this problem (i.e. without trimming; see Figure 1a) by imposing, for example, that $x_{i k \ell}=0$ if $w_{i} \neq w_{k}$ in model $\mathcal{M}_{1}^{L}$, and that $x_{i k \ell}=0$ if $w_{i} \neq \bar{w}_{\beta_{k}}$ in model $\mathcal{M}_{2}^{L}$, or simply removing these variables from the models. Moreover, both models can also be modified to deal with cases in which the items can be rotated by 90 degrees in the cutting/packing patterns. This can be done in model $\mathcal{M}_{1}^{L}$ by introducing for each item $i$ a counterpart item $i^{\prime}$ for which its width and height are defined as $w_{i^{\prime}}=h_{i}$ and $h_{i^{\prime}}=w_{i}$ (i.e. the dimensions of items $i$ and $i^{\prime}$ are swapped) and by adding to the formulation constraints to avoid that items $i$ and $i^{\prime}$ are both produced from the objects cut. A similar reasoning can also be applied to the item types of model $\mathcal{M}_{2}^{L}$.

\section{Numerical experiments}

In this section we present and analyze some numerical experiments with models $\mathcal{M}_{1}^{L}$ and $\mathcal{M}_{2}^{L}$. Twenty arbitrary randomly-generated small-scale instances were considered to illustrate both models. It is worth noting that medium and large-scale instances available in the literature are not suitable to be solved, by the proposed models, with an exact method such as CPLEX. The first ten instances have strongly heterogeneous items, being representatives of the residual bin-packing problem, while the second set of ten instances corresponds to weakly heterogeneous items and represents the residual cutting-stock problem. Table 1 describes the objects and items that compose each instance. In the table, $p$ is the number of objects and $n$ is the number of items. From the data in the table, it is straightforward to obtain the data to build the instances for models $\mathcal{M}_{1}^{L}$ and $\mathcal{M}_{2}^{L}$ (that consider the items individually and grouped by type, respectively). In all instances, we considered $c_{\ell}=\bar{c}_{\ell}=1$ for all $\ell$. For each instance, we also considered $d_{\text {min }}$ equal to the smallest height of the instance' demanded items. Table 2 displays the number of continuous, binary, and integer variables and the number of constraints of the twenty considered instances of models $\mathcal{M}_{1}^{L}$ and $\mathcal{M}_{2}^{L}$. In the models, we always considered big- $M$ constants $M_{\ell} \equiv H_{\ell}$ and $\bar{M}_{\ell} \equiv H_{\ell} W_{\ell}$ for $\ell=1, \ldots, p$.

MIP models $\mathcal{M}_{1}^{L}$ and $\mathcal{M}_{2}^{L}$ were implemented in $\mathrm{C} / \mathrm{C}++$ using the ILOG Concert Technology 2.9 and compiled with $\mathrm{g}++$ from gcc version 4.6.1 (GNU compiler collection). Numerical experiments were conducted on a machine with two $2.67 \mathrm{GHz}$ Intel Xeon CPU X5650 processors, 8GB of RAM memory, and running GNU/Linux operating system (Ubuntu 12.04 LTS, kernel 3.2.0-33). Instances were solved using the branch-and-cut method in IBM ILOG CPLEX 12.1.0. By default, a solution is reported as optimal by the solver when

$$
\text { absolute gap }=\text { best feasible solution }- \text { best lower bound } \leq \varepsilon_{\text {abs }}
$$

or

$$
\text { relative gap }=\frac{\mid \text { best feasible solution }- \text { best lower bound } \mid}{1 \mathrm{e}-10+\mid \text { best feasible solution } \mid} \leq \varepsilon_{\text {rel }},
$$

with $\varepsilon_{\text {abs }}=10^{-6}$ and $\varepsilon_{\text {rel }}=10^{-4}$, where "best feasible solution" means the smallest value of the objective function related to a feasible solution generated by the method. Since decimal places of the objective functions (62) and (77) of models $\mathcal{M}_{1}^{L}$ and $\mathcal{M}_{2}^{L}$, respectively, represent the value of the leftovers, we inhibited the relative gap stopping criterion setting $\varepsilon_{\text {rel }}=0$, to avoid stopping the method prematurely. All other parameters of the solver were used with their default values unless otherwise stated.

Table 3 describes the solutions to the twenty instances associated with models $\mathcal{M}_{1}^{L}$ and $\mathcal{M}_{2}^{L}$. In the table, "Optimal value" corresponds to the value of the objective function at the solution 


\begin{tabular}{|c|c|c|c|c|}
\hline \multirow{2}{*}{ Inst. } & \multicolumn{2}{|r|}{ Objects } & \multicolumn{2}{|r|}{ Items } \\
\hline & $p$ & width $\times$ height & $n$ & width $\times$ height \\
\hline 1 & 3 & $3(52 \times 53)$ & 32 & $\begin{array}{l}19 \times 21,7 \times 20,4 \times 20,15 \times 20,14 \times 20,14 \times 19, \\
17 \times 19,10 \times 17,13 \times 17,5 \times 17,16 \times 17,20 \times 16, \\
5 \times 16,3 \times 15,5 \times 14,18 \times 14,10 \times 13,14 \times 12, \\
11 \times 11,2 \times 10,7 \times 9,14 \times 8,13 \times 8,9 \times 7, \\
7 \times 7,16 \times 6,20 \times 5,2 \times 5,9 \times 3,17 \times 3,5 \times 3, \\
4 \times 2\end{array}$ \\
\hline 2 & 2 & $2(63 \times 60)$ & 23 & $\begin{array}{l}22 \times 23,17 \times 23,13 \times 22,7 \times 22,9 \times 21,5 \times 20, \\
20 \times 20,6 \times 19,7 \times 17,14 \times 16,12 \times 14,15 \times 14, \\
20 \times 14,9 \times 10,16 \times 9,20 \times 9,18 \times 8,3 \times 8, \\
12 \times 7,18 \times 6,20 \times 4,9 \times 3,6 \times 3\end{array}$ \\
\hline 3 & 7 & $24 \times 14,2(18 \times 10), 24 \times 13,3(13 \times 10)$ & 17 & $\begin{array}{l}3 \times 5,2 \times 5,4 \times 3,6 \times 3,3 \times 3,7 \times 3,2 \times 2, \\
6 \times 2,9 \times 2,4 \times 2,1 \times 2,7 \times 2,4 \times 1,6 \times 1, \\
2 \times 1,9 \times 1,7 \times 1\end{array}$ \\
\hline 4 & 11 & $2(24 \times 14), 3(18 \times 10), 2(24 \times 13), 4(13 \times 10)$ & 27 & $\begin{array}{l}3 \times 5,1 \times 5,7 \times 5,2 \times 5,3 \times 4,7 \times 4,4 \times 4, \\
4 \times 3,1 \times 3,6 \times 3,8 \times 3,3 \times 3,7 \times 3,5 \times 2, \\
6 \times 2,2 \times 2,9 \times 2,4 \times 2,1 \times 2,7 \times 2,8 \times 1, \\
2 \times 1,6 \times 1,7 \times 1,9 \times 1,4 \times 1,5 \times 1\end{array}$ \\
\hline 5 & 16 & $2(24 \times 14), 5(18 \times 10), 3(24 \times 13), 6(13 \times 10)$ & 37 & $\begin{array}{l}3 \times 5,1 \times 5,7 \times 5,2 \times 5,8 \times 5,6 \times 4,5 \times 4, \\
3 \times 4,7 \times 4,4 \times 4,1 \times 4,2 \times 4,4 \times 3,5 \times 3, \\
1 \times 3,2 \times 3,6 \times 3,8 \times 3,3 \times 3,7 \times 3,6 \times 2, \\
9 \times 2,7 \times 2,4 \times 2,5 \times 2,1 \times 2,2 \times 2,3 \times 2, \\
6 \times 1,2 \times 1,4 \times 1,8 \times 1,5 \times 1,9 \times 1,3 \times 1, \\
1 \times 1,7 \times 1\end{array}$ \\
\hline 6 & 8 & $2(24 \times 14), 4(18 \times 10), 2(24 \times 13)$ & 34 & $\begin{array}{l}3 \times 5,1 \times 5,7 \times 5,2 \times 5,8 \times 5,6 \times 4,3 \times 4, \\
7 \times 4,4 \times 4,1 \times 4,2 \times 4,4 \times 3,5 \times 3,1 \times 3, \\
6 \times 3,8 \times 3,3 \times 3,7 \times 3,6 \times 2,7 \times 2,9 \times 2, \\
5 \times 2,4 \times 2,2 \times 2,1 \times 2,3 \times 2,9 \times 1,7 \times 1, \\
8 \times 1,5 \times 1,2 \times 1,6 \times 1,4 \times 1,1 \times 1\end{array}$ \\
\hline 7 & 3 & $24 \times 14,18 \times 10,24 \times 13$ & 11 & $\begin{array}{l}2(2 \times 2), 6 \times 2,4 \times 2,1 \times 2,2(7 \times 1), 4 \times 1, \\
9 \times 1,6 \times 1,2 \times 1\end{array}$ \\
\hline 8 & 10 & $3(28 \times 17), 3(16 \times 27), 4(13 \times 23)$ & 19 & $\begin{array}{l}1 \times 10,6 \times 10,4 \times 9,8 \times 9,9 \times 9,5 \times 8,2 \times 7, \\
4 \times 6,10 \times 6,6 \times 6,10 \times 5,5 \times 5,8 \times 5,4 \times 4, \\
7 \times 4,10 \times 4,6 \times 4,7 \times 3,10 \times 2\end{array}$ \\
\hline 9 & 4 & $3(19 \times 10), 19 \times 26$ & 17 & $\begin{array}{l}2 \times 9,2 \times 8,5 \times 8,3 \times 8,4 \times 7,5 \times 7,4 \times 6, \\
6 \times 4,2 \times 4,3 \times 4,4 \times 3,2 \times 3,6 \times 2,5 \times 2, \\
1 \times 1,2 \times 1,3 \times 1\end{array}$ \\
\hline 10 & 6 & $2(290 \times 106), 2(148 \times 183), 2(194 \times 132)$ & 13 & $\begin{array}{l}2(63 \times 59), 63 \times 55,48 \times 48,17 \times 43,98 \times 40, \\
38 \times 35,2(114 \times 33), 24 \times 23,62 \times 19,2(110 \times 11)\end{array}$ \\
\hline 11 & 13 & $5(25 \times 21), 5(27 \times 19), 3(30 \times 24)$ & 37 & $8(11 \times 7), 5(7 \times 5), 9(9 \times 5), 5(10 \times 5), 10(3 \times 2)$ \\
\hline 12 & 1 & $14 \times 19$ & 12 & $12(2 \times 4)$ \\
\hline 13 & 7 & $2(21 \times 24), 5(10 \times 18)$ & 32 & $15(4 \times 7), 17(1 \times 4)$ \\
\hline 14 & 4 & $24 \times 14,2(18 \times 10), 24 \times 13$ & 28 & $12(7 \times 1), 7(6 \times 1), 9(4 \times 1)$ \\
\hline 15 & 12 & $4(26 \times 19), 4(22 \times 23), 4(30 \times 17)$ & 34 & $13(7 \times 6), 10(9 \times 4), 11(11 \times 3)$ \\
\hline 16 & 19 & $6(30 \times 11), 6(27 \times 13), 7(12 \times 26)$ & 21 & $10(8 \times 10), 9(10 \times 3), 2(11 \times 2)$ \\
\hline 17 & 1 & $14 \times 19$ & 17 & $7(2 \times 4), 10(1 \times 3)$ \\
\hline 18 & 5 & $3(22 \times 17), 2(14 \times 30)$ & 24 & $14(2 \times 11), 10(5 \times 5)$ \\
\hline 19 & 16 & $4(30 \times 22), 4(30 \times 24), 8(10 \times 21)$ & 41 & $15(9 \times 7), 11(11 \times 6), 15(1 \times 5)$ \\
\hline 20 & 9 & $5(22 \times 17), 4(14 \times 30)$ & 21 & $8(2 \times 11), 7(8 \times 9), 6(5 \times 5)$ \\
\hline
\end{tabular}

Table 1: Description of the considered instances. Dimensions are given in the format width $x$ height. Notation $a(b \times c)$ means that there are $a$ objects or items with dimension $b \times c$. When $a$ is omitted it means that there is a single copy of the described object or item. 


\begin{tabular}{|r|rrr|rrrr|}
\hline \multirow{2}{*}{ Inst. } & \multicolumn{3}{|c|}{ Model $\mathcal{M}_{1}^{L}$} & \multicolumn{4}{c|}{ Model $\mathcal{M}_{2}^{L}$} \\
\cline { 2 - 7 } & $\begin{array}{c}\text { Continuous } \\
\text { variables }\end{array}$ & $\begin{array}{c}\text { Binary } \\
\text { variables }\end{array}$ & $\begin{array}{c}\text { Constraints } \\
\text { Continuous } \\
\text { variables }\end{array}$ & $\begin{array}{c}\text { Binary } \\
\text { variables }\end{array}$ & $\begin{array}{c}\text { Integer } \\
\text { variables }\end{array}$ & Constraints \\
\hline 1 & 6 & 1,590 & 149 & 6 & 102 & 1,584 & 1,797 \\
2 & 4 & 556 & 83 & 4 & 50 & 552 & 681 \\
3 & 14 & 1,084 & 185 & 14 & 133 & 1,071 & 1,290 \\
4 & 22 & 4,179 & 401 & 22 & 319 & 4,158 & 4,613 \\
5 & 32 & 11,280 & 741 & 32 & 624 & 11,248 & 12,063 \\
6 & 16 & 4,776 & 362 & 16 & 288 & 4,760 & 5,190 \\
7 & 6 & 203 & 69 & 6 & 39 & 177 & 266 \\
8 & 20 & 1,920 & 279 & 20 & 210 & 1,900 & 2,217 \\
9 & 8 & 620 & 113 & 8 & 76 & 612 & 759 \\
10 & 12 & 558 & 139 & 12 & 90 & 420 & 582 \\
11 & 26 & 9,164 & 673 & 26 & 507 & 1,391 & 2,106 \\
12 & 2 & 80 & 53 & 2 & 14 & 12 & 78 \\
13 & 14 & 3,710 & 365 & 14 & 238 & 329 & 728 \\
14 & 8 & 1,632 & 218 & 8 & 120 & 236 & 485 \\
15 & 24 & 7,164 & 588 & 24 & 432 & 840 & 1,465 \\
16 & 38 & 4,426 & 589 & 38 & 437 & 950 & 1,563 \\
17 & 2 & 154 & 71 & 2 & 19 & 24 & 114 \\
18 & 10 & 1,510 & 223 & 10 & 130 & 190 & 439 \\
19 & 32 & 13,808 & 885 & 32 & 688 & 1,312 & 2,241 \\
20 & 18 & 2,096 & 309 & 18 & 207 & 396 & 729 \\
\hline
\end{tabular}

Table 2: Number of continuous, binary, and integer variables and number of constraints of the twenty considered instances of models $\mathcal{M}_{1}^{L}$ and $\mathcal{M}_{2}^{L}$.

reported by the solver as optimal. "Objects cost" and "Leftovers value" correspond to the cost of the used objects at the optimal solution and the value of the leftovers, respectively. Those values are extracted from the optimal value according to (62) and (77) for models $\mathcal{M}_{1}^{L}$ and $\mathcal{M}_{2}^{L}$, respectively. Remaining columns "MIP iterations", "B\&B Nodes", and "CPU time" (in seconds) are self-explanatory and state the effort required by the solver to obtain the reported solution. As illustrative examples, Figures 4-8 show the graphical representation (cutting/packing patterns) of the solutions obtained (considering model $\mathcal{M}_{2}^{L}$ ) for the arbitrarily selected instances 1, 6, 10, 16, and 20. In the figures, dashed regions represent leftovers while blank spaces correspond to waste. The graphical representation of the solutions obtained for the whole set of instances can be found in Andrade et al. (2013).

From the results of Table 3, it is possible to see that there is no clear winner in the first half of the instances set, while model $\mathcal{M}_{2}^{L}$ is clearly easier to be solved in the second half of the instances set, as expected. The stopping criterion was satisfied in 19 cases (all but instance 11 for which the solver ran out of memory) for model $\mathcal{M}_{1}^{L}$ and in all cases for model $\mathcal{M}_{2}^{L}$. The best feasible solution reported for instance 11 by model $\mathcal{M}_{1}^{L}$ coincides with the optimal one found when solving model $\mathcal{M}_{2}^{L}$. Instances that combine a large number $p$ of objects with a large number $n$ of items (like instances 4, 5, 11, and 15; see Table 1) appear to be the hardest ones when a branch-and-cut exact method like CPLEX is used. This may be considered an expected consequence of the number of binary variables of the models, which depends on $p$ and $n$ (model $\mathcal{M}_{1}^{L}$ has $2 p+n p / 2+n 2 / 2$ binary variables, while model $\mathcal{M}_{2}^{L}$ has $2 p+n p$ binary variables; see Section 3.1 ). The bottleneck for considering instances larger than the ones presented in this experiments lies on the usage of memory. On larger instances the solver ran out of memory (as it was the case of instance 11 of model $\mathcal{M}_{1}^{L}$ ) if only primary (RAM) memory is used. When considering secondary memory (files on disk), the time for swapping is unaffordable. This observation supports the necessity for developing 


\begin{tabular}{|c|r|rr|rrr|}
\hline \multicolumn{7}{|c|}{ Model $\mathcal{M}_{1}^{L}$} \\
\hline \multirow{2}{*}{ Inst. } & Optimal & Salue & Solutions description & \multicolumn{3}{c|}{ Effort measurements } \\
\cline { 3 - 7 } & Objects cost & Leftovers value & MIP Iterations & B\&B Nodes & CPU Time \\
\hline \hline 1 & $5,511.937107$ & 5,512 & 520 & $10,778,938$ & 649,747 & 171.06 \\
2 & $7,559.616667$ & 7,560 & 2,898 & 42,798 & 2,079 & 1.23 \\
3 & 259.962804 & 260 & 52 & 146,570 & 7,040 & 1.88 \\
4 & 359.999998 & 360 & 0 & $55,955,833$ & 1701,516 & $1,286.04$ \\
5 & 466.000000 & 466 & 0 & $174,832,220$ & 1508,414 & $8,752.14$ \\
6 & 491.976187 & 492 & 48 & $22,395,534$ & 632,600 & 451.53 \\
7 & 179.869565 & 180 & 108 & 317 & 9 & 0.07 \\
8 & 863.983673 & 864 & 64 & $1,349,127$ & 28,641 & 30.07 \\
9 & 380.000000 & 380 & 0 & 25,848 & 1,227 & 1.50 \\
10 & $51,215.922104$ & 51,216 & 12,998 & 5,522 & 189 & 0.29 \\
11 & $1,745.9918 *$ & 1,746 & 60 & $40,810,461$ & 335,600 & $2,319.25$ \\
12 & 265.421053 & 266 & 154 & 44 & 0 & 0.00 \\
13 & 683.947589 & 684 & 100 & 132,993 & 1,605 & 7.43 \\
14 & 179.982143 & 180 & 18 & 17,937 & 383 & 0.67 \\
15 & $1,506.000000$ & 1,506 & 0 & $93,466,859$ & 2033,696 & $2,981.30$ \\
16 & $1,364.994258$ & 1,365 & 36 & 203,713 & 4,285 & 7.46 \\
17 & 265.368421 & 266 & 168 & 92 & 0 & 0.03 \\
18 & 748.000000 & 748 & 0 & 4,559 & 58 & 0.38 \\
19 & $2,010.000000$ & 2,010 & 132 & 320,459 & 3,411 & 43.92 \\
20 & $1,167.962817$ & 1,168 & & & 7.98 \\
\hline
\end{tabular}

\begin{tabular}{|c|c|c|c|c|c|c|}
\hline \multicolumn{7}{|c|}{ Model $\mathcal{M}_{2}^{L}$} \\
\hline \multirow{2}{*}{ Inst. } & \multirow{2}{*}{$\begin{array}{l}\text { Optimal } \\
\text { value }\end{array}$} & \multicolumn{2}{|c|}{ Solutions description } & \multicolumn{3}{|c|}{ Effort measurements } \\
\hline & & Objects cost & Leftovers value & MIP Iterations & B\&B Nodes & CPU Time \\
\hline 1 & $5,511.937107$ & 5,512 & 520 & $10,833,135$ & 659,436 & 171.35 \\
\hline 2 & $7,559.616667$ & 7,560 & 2,898 & 78,562 & 3,088 & 1.51 \\
\hline 3 & 259.962804 & 260 & 52 & 146,570 & 7,040 & 1.90 \\
\hline 4 & 360.000000 & 360 & 0 & $13,905,664$ & 215,651 & 247.56 \\
\hline 5 & 466.000000 & 466 & 0 & $167,435,459$ & $1,573,589$ & $10,138.93$ \\
\hline 6 & 491.976189 & 492 & 48 & $24,503,629$ & 713,002 & 514.95 \\
\hline 7 & 179.869565 & 180 & 108 & 356 & 0 & 0.04 \\
\hline 8 & 863.983673 & 864 & 64 & 873,289 & 16,808 & 12.68 \\
\hline 9 & 380.000000 & 380 & 0 & 25,848 & 1,227 & 1.50 \\
\hline 10 & $51,215.922104$ & 51,216 & 12,998 & 4,332 & 165 & 0.26 \\
\hline 11 & $1,745.991836$ & 1,746 & 60 & $24,461,056$ & 794,785 & 602.00 \\
\hline 12 & 265.421053 & 266 & 154 & 29 & 0 & 0.03 \\
\hline 13 & 683.947589 & 684 & 100 & 4,163 & 173 & 0.28 \\
\hline 14 & 179.982143 & 180 & 18 & 17,766 & 951 & 0.38 \\
\hline 15 & $1,505.999996$ & 1,506 & 0 & $52,485,990$ & 1105,225 & $2,298.26$ \\
\hline 16 & $1,364.994258$ & 1,365 & 36 & 72,094 & 3,491 & 3.55 \\
\hline 17 & 265.368421 & 266 & 168 & 78 & 0 & 0.00 \\
\hline 18 & 748.000000 & 748 & 0 & 1,963 & 71 & 0.17 \\
\hline 19 & $2,010.000000$ & 2,010 & 0 & 116,686 & 3,161 & 5.79 \\
\hline 20 & $1,167.962817$ & 1,168 & 132 & 78,712 & 2,974 & 2.57 \\
\hline
\end{tabular}

Table 3: Numerical results for the twenty instances of models $\mathcal{M}_{1}^{L}$ and $\mathcal{M}_{2}^{L}$. ${ }^{*}$ Value reported for instance 11 of model $\mathcal{M}_{1}^{L}$ in the column "Optimal value" correspond to the best feasible solution found, since the solver ran out of memory and the absolute gap stopping criterion was not satisfied. The reported best lower bound was 1,745.9799.

dedicated exact and heuristic method to tackle the introduced problems. 

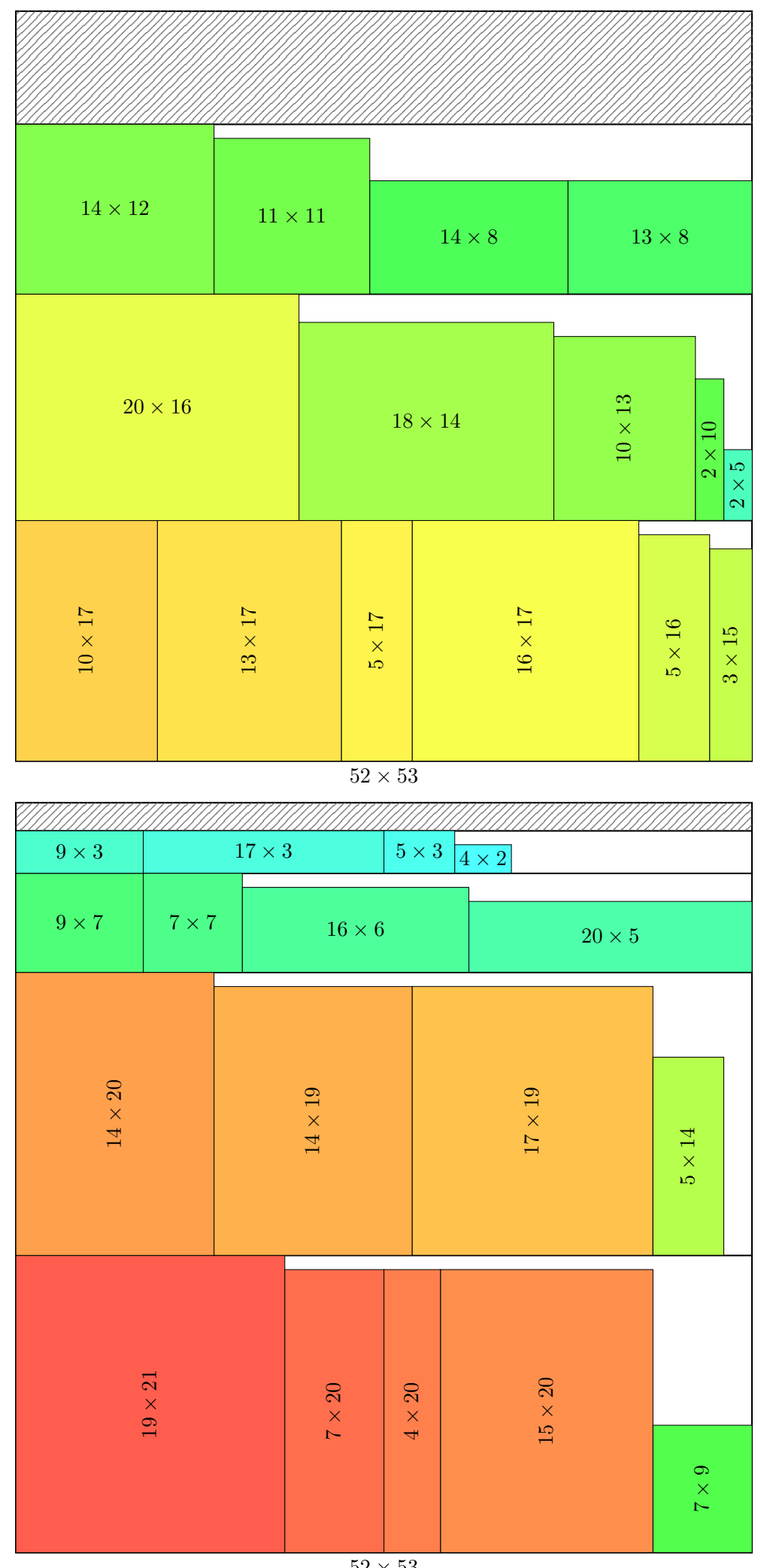

Figure 4: Graphical representation of the solution to instance 1. 


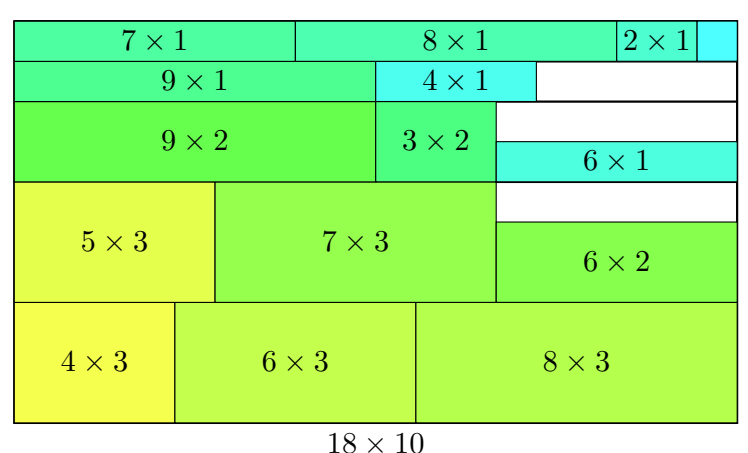

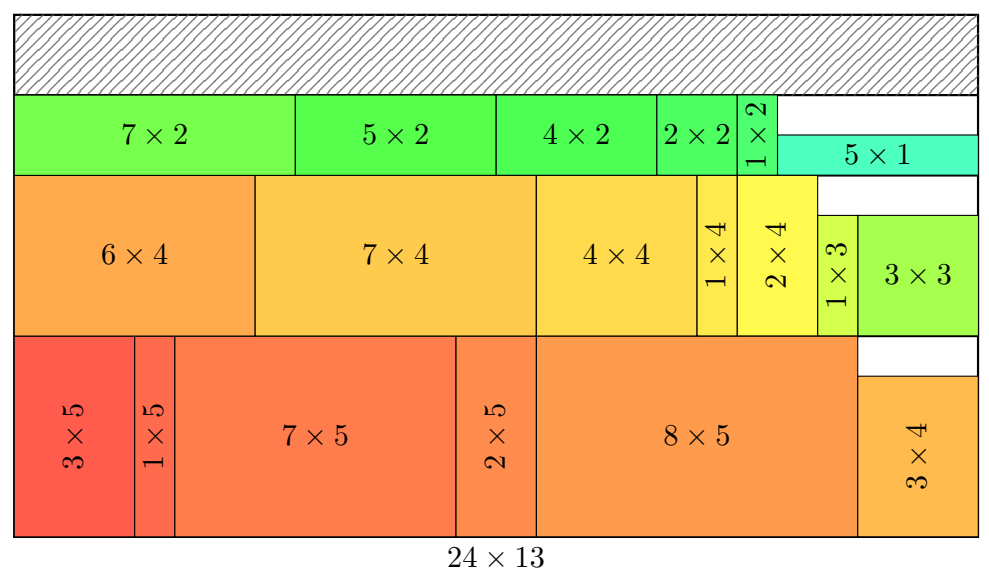

Figure 5: Graphical representation of the solution to instance 6.
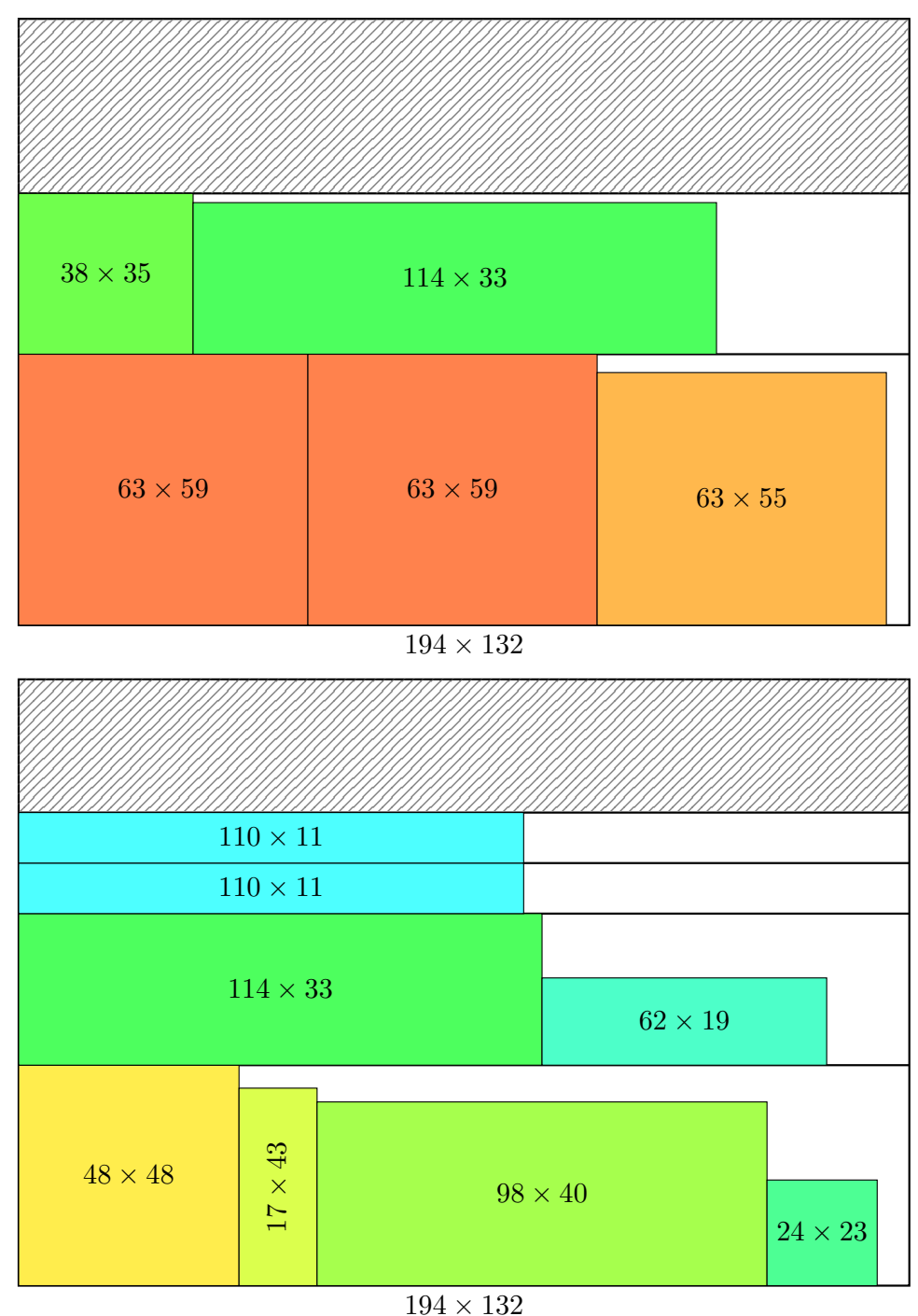

Figure 6: Graphical representation of the solution to instance 10. 

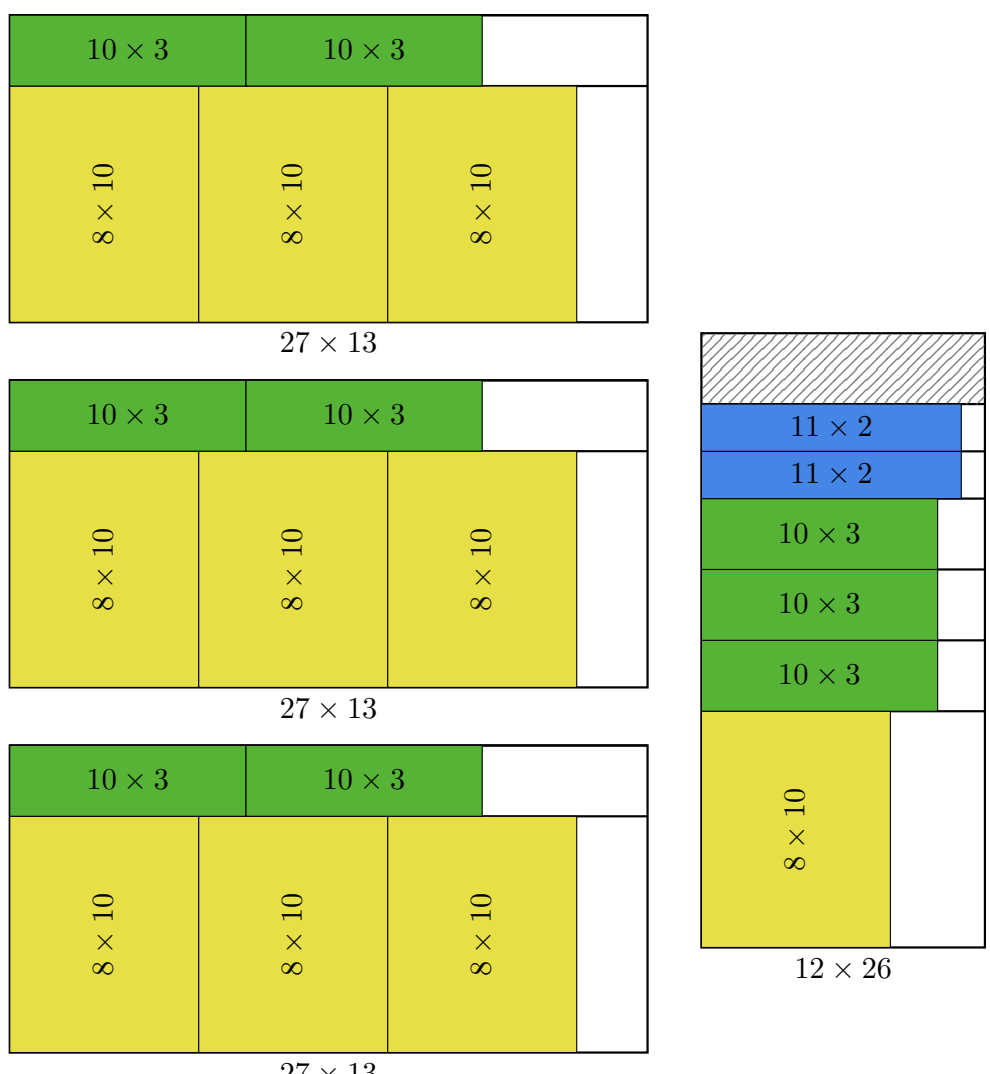

Figure 7: Graphical representation of the solution to instance 16.
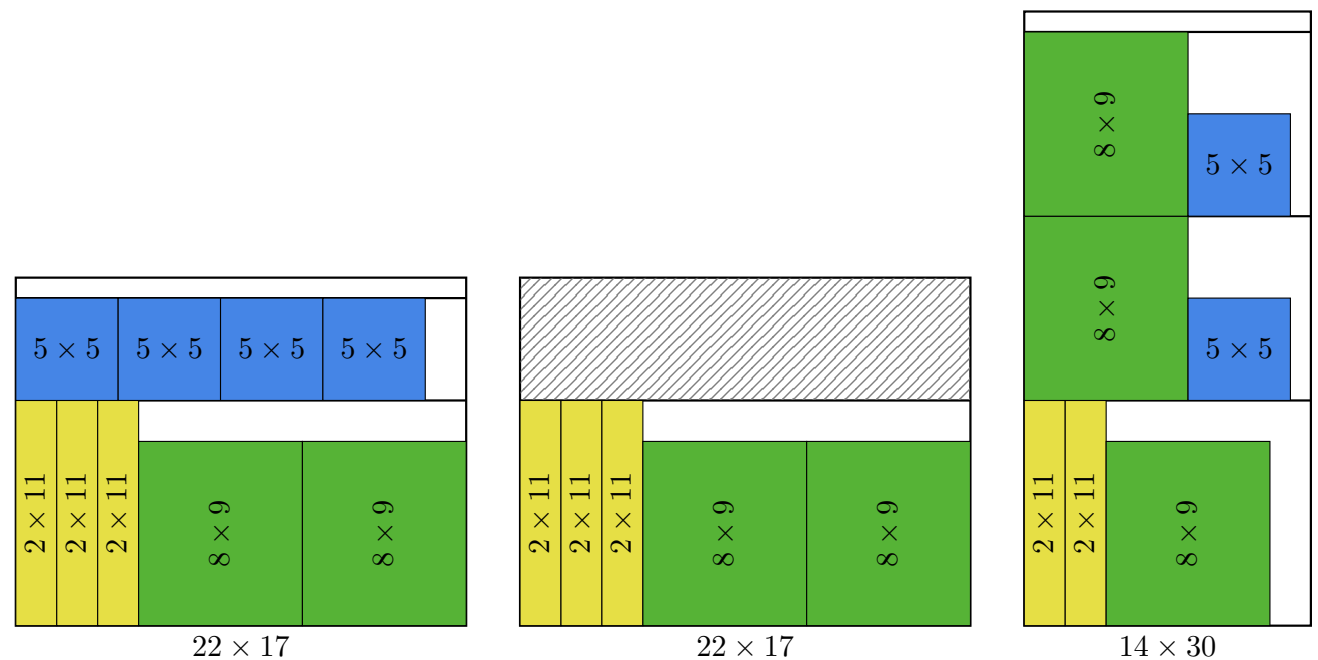

Figure 8: Graphical representation of the solution to instance 20. 


\section{Conclusions}

In this study we presented two MIP models for the non-exact two-stage two-dimensional guillotine cutting/packing problem with usable objects remainders. Both models are based on bilevel mathematical programming formulations for the problem, but because of special characteristics of these bilevel models, they can be reformulated as one-level MIP models. The models can be modified to deal with particular cases of the tackled problem, such as the exact two-stage guillotine cutting without trimming, and with more general cases, such as the case in which the items can be rotated and the case in which the first-stage cuts on the plates can be either horizontal (parallel to the plate width) or vertical (parallel to the plate height). To the best of our knowledge, there are no other studies in the literature dealing with two-stage two-dimensional guillotine cutting/packing problems with usable leftovers. Numerical experiments illustrating the models and their scope and limitations were presented. The formal definition of these variants of two-dimensional guillotine cutting problems with residual pieces opens up interesting possibilities for the development of dedicated exact and heuristic methods for the resolution and practical application of these and other cutting/packing problems of two and more dimensions as, for example, the two-dimensional non-guillotine cutting/packing problem with usable leftovers.

\section{References}

Abuabara, A. \& Morabito, R. (2009), 'Cutting optimization of structural tubes to build agricultural light aircrafts', Annals of Operations Research 169(1), 149-165.

Alvarez-Valdes, R., Parajon, A. \& Tamarit, J. M. (2002), 'A computational study of LPbased heuristic algorithms for two-dimensional guillotine cutting stock problems', OR Spektrum 24(2), 179-192.

Andrade, R., Birgin, E. G. \& Morabito, R. (2013), Two-stage two-dimensional guillotine cutting problems with usable leftovers, Technical Report MCDO15113, Department of Computer Science, Institute of Mathematics and Statistics, University of São Paulo, Brazil.

URL: http://www.ime.usp.br/ egbirgin/

Andrade, R., Birgin, E. G., Morabito, R. \& Ronconi, D. P. (2014), 'MIP models for two-dimensional non-guillotine cutting problems with usable leftovers', Journal of the Operational Research Society .

Bang-Jensen, J. \& Larsen, R. (2012), 'Efficient algorithms for real-life instances of the variable size bin packing problem', Computers \& Operations Research 39(11), 2848-2857.

Beasley, J. E. (1985), 'Algorithms for unconstrained two-dimensional guillotine cutting', Journal of the Operational Research Society 36(4), 297-306.

Belov, G. \& Scheithauer, G. (2006), 'A branch-and-cut-and-price algorithm for one-dimensional stock cutting and two-dimensional two-stage cutting', European Journal of Operational Research 171(1), 85-106.

Brown, A. R. (1971), Optimum packing and depletion: the computer in space- and resource-usage problem, Macdonald and Co., London.

Carnieri, C., Mendoza, G. A. \& Gavinho, L. G. (1994), 'Solution procedures for cutting lumber into furniture parts', European Journal of Operational Research 73(3), 495-501. 
Cherri, A. C., Arenales, M. N. \& Yanasse, H. H. (2009), 'The one-dimensional cutting stock problem with usable leftover - A heuristic approach', European Journal of Operational Research 196(1), 897-908.

Cherri, A. C., Arenales, M. N. \& Yanasse, H. H. (2013), 'The usable leftover one-dimensional cutting stock problem - A priority-in-use heuristic', International Transactions in Operational Research 20(2), 189-199.

Cintra, G. F., Miyazawa, F. K., Wakabayashi, Y. \& Xavier, E. C. (2008), 'Algorithms for twodimensional cutting stock and strip packing problems using dynamic programming and column generation', European Journal of Operational Research 191(1), 61-85.

Cui, Y. (2013), 'A new dynamic programming procedure for three-staged cutting patterns', Journal of Global Optimization 55(2), 349-357.

Cui, Y. \& Huang, B. (2012), 'Reducing the number of cuts in generating three-staged cutting patterns', European Journal of Operational Research 218(2), 358-365.

Cui, Y., Wang, Z. \& Li, J. (2005), 'Exact and heuristic algorithms for staged cutting problems', Proceedings of the Institution of Mechanical Engineers, Part B: Journal of Engineering Manufacture 219(2), 201-207.

Cui, Y. \& Yang, Y. (2010), 'A heuristic for the one-dimensional cutting stock problem with usable leftover', European Journal of Operational Research 204(2), 245-250.

Cui, Y. \& Zhao, Z. (2013), 'Heuristic for the rectangular two-dimensional single stock size cutting stock problem with two-staged patterns', European Journal of Operational Research 231(2), 288298.

Dempe, S. (2002), Foundations of bilevel programming, Springer, Dordrecht.

Dimitriadis, S. \& Kehris, E. (2009), 'Cutting stock process optimisation in custom door and window manufacturing industry', International Journal of Decision Sciences, Risk and Management $\mathbf{1}(1), 66-80$.

Dyckhoff, H. (1981), 'A new linear programming approach to the cutting stock problem', Operations Research 29(6), 1092-1104.

Farley, A. (1983), 'Practical adaptations of the Gilmore-Gomory approach to cutting stock problems', OR Spektrum 10(2), 113-123.

Furini, F. \& Malaguti, E. (2013), 'Models for the two-dimensional two-stage cutting stock problem with multiple stock size', Computers \& Operations Research 40(8), 1953-1962.

Furini, F., Malaguti, E., Durán, R. M., Persiani, A. \& Toth, P. (2012), 'A column generation heuristic for the two-dimensional two-staged guillotine cutting stock problem with multiple stock size', European Journal of Operational Research 218(1), 251-260.

Gilmore, P. C. \& Gomory, R. E. (1965), 'Multistage cutting stock problems of two and more dimensions', Operations Research 13(1), 94-120.

Gradisar, M., Erjavec, J. \& Tomat, L. (2011), 'One-dimensional cutting stock optimization with usable leftover: A case of low stock-to-order ratio', International Journal of Decision Support System Technology 3(1), 54-66. 
Gradisar, M., Jesenko, J. \& Resinovic, C. (1997), 'Optimization of roll cutting in clothing industry', Computers $\&$ Operational Research 24(10), 945-953.

Gradisar, M., Kljajic, M., Resinovic, C. \& Jesenko, J. (1999), 'A sequential heuristic procedure for one-dimentional cutting', European Journal of Operational Research 114(3), 557-568.

Gradisar, M. \& Trkman, P. (2005), 'A combined approach to the solution to the general onedimensional cutting stock problem', Computers \& Operations Research 32(7), 1793-1807.

Hadjiconstantinou, E. \& Christofides, N. (1995), 'An exact algorithm for general, orthogonal, twodimensional knapsack problems', European Journal of Operational Research 83(1), 39-56.

Hifi, M. (1997), 'The DH/KD algorithm: A hybrid approach for unconstrained two-dimensional cutting problems', European Journal of Operational Research 97(1), 41-52.

Hifi, M. \& Roucairol, C. (2001), 'Approximate and exact algorithms for constrained (un)weighted two-dimensional two-staged cutting stock problems', Journal of Combinatorial Optimization 5(4), 465-494.

Hinxman, A. I. (1980), 'The trim-loss and assortment problems: A survey', European Journal of Operational Research 5(1), 8-18.

Koch, S., König, S. \& Ws̈cher, G. (2009), 'Integer linear programming for a cutting problem in the wood-processing industry: a case study', International Transactions in Operational Research 16(6), 715-726.

Lodi, A., Martello, S. \& Monaci, M. (2002), 'Two-dimensional packing problems: a survey', European Journal of Operational Research 141(2), 241-252.

Lodi, A. \& Monaci, M. (2003), 'Integer linear programming models for 2-staged two-dimensional knapsack problems', Mathematical Programming 94(2-3), 257-278.

Macedo, R., Alves, C. \& Valério de Carvalho, J. M. (2010), 'Arc-flow model for the two-dimensional guillotine cutting stock problem', Computers \& Operations Research 37(6), 991-1001.

Morabito, R. \& Arenales, M. N. (1996), 'Staged and constrained two-dimensional guillotine cutting problems: An and/or-graph approach', European Journal of Operational Research 94(3), 548560 .

Morabito, R. \& Arenales, M. N. (2000), 'Optimizing the cutting of stock plates in a furniture company', International Journal of Production Research 38(12), 2725-2742.

Morabito, R. \& Belluzzo, L. (2005), 'Otimização nos padrões de corte de chapas de fibra de madeira reconstituída: um estudo de caso', Pesquisa Operacional 25(3), 391-415.

Morabito, R. \& Garcia, V. (1998), 'The cutting stock problem in a hardboard industry: A case study', Computers \& Operations Research 25(6), 469-485.

Morabito, R. \& Pureza, V. (2010), 'A heuristic approach based on dynamic programming and and/or-graph search for the constrained two-dimensional guillotine cutting problem', Annals of Operations Research 179, 297-315.

Mrad, M., Meftahi, I. \& Haouari, M. (2013), 'A branch-and-price algorithm for the two-stage guillotine cutting stock problem', Journal of the Operational Research Society 64(5), 629-637. 
Poldi, K. C. \& Arenales, M. N. (2006), 'Heurísticas para o problema de corte de estoque unidimensional inteiro', Pesquisa Operacional 26(3), 473-492.

Poldi, K. C. \& Arenales, M. N. (2009), 'Heuristics for the one-dimensional cutting stock problem with limited multiple stock lengths', Computers $\&$ Operations Research 36(6), 2074-2081.

Riehme, J., Scheithauer, G. \& Terno, J. (1996), 'The solution of two-stage guillotine cutting stock problems having extremely varying order demands', European Journal of Operational Research 91(3), 543-552.

Roodman, G. M. (1986), 'Near-optimal solutions to one-dimensional cutting stock problems', Computers and Operations Research 13(6), 713-719.

Scheithauer, G. (1991), 'A note on handling residual lengths', Optimization 22(3), 461-466.

Silva, E., Alvelos, F. \& Valério de Carvalho, J. M. (2010), 'An integer programming model for two- and three-stage two-dimensional cutting stock problems', European Journal of Operational Research 205(3), 699-708.

Sinuany-Stern, Z. \& Weiner, I. (1994), 'The one dimensional cutting stock problem using two objectives', Journal of Operations Research Society 45(2), 231-236.

Trkman, P. \& Gradisar, M. (2007), 'One-dimensional cutting stock optimization in consecutive time periods', European Journal of Operational Research 179(2), 291-301.

Wäscher, G. \& Gau, T. (1996), 'Heuristics for the integer one-dimensional cutting stock problem: A computational study', OR-Spektrum 18, 131-144.

Wäscher, G., Haußner, H. \& Schumann, H. (2007), 'An improved typology of cutting and packing problems', European Journal of Operational 183(3), 1109-1130.

Yanasse, H. H. \& Katsurayama, D. M. (2005), 'Checkerboard pattern: proposals for its generation', International Transactions in Operational Research 12(1), 21-45.

Yanasse, H. H., Zinober, A. \& Harris, R. (1991), 'Two-dimensional cutting stock with multiple stock sizes', Journal of the Operational Research Society 42(8), 673-683. 